\title{
MODELLING A PEROXIDASE-BASED FLUORESCENT BIOSENSOR
}

\author{
Evelina Gaidamauskaitè \\ Department of Software Engineering \\ Vilnius University \\ Naugarduko str. 24, LT-03225, Vilnius, Lithuania \\ E-mail: evelina.gaidamauskaite@mif.vu.lt
}

\author{
Romas Baronas \\ Institute of Mathematics and Informatics \\ Vilnius University \\ Akademijos str. 4, LT-08663, Vilnius, Lithuania \\ E-mail: romas.baronas@mif.vu.lt
}

\section{KEYWORDS}

Modelling, reaction-diffusion, simulation, fluorescent biosensor.

\begin{abstract}
The paper presents a one-dimensional-in-space mathematical model of a peroxidase-based fluorescent biosensor. The mathematical model is based on a system of nonlinear reaction diffusion equations. The problem was solved numerically using finite difference technique. The model was used to study the effect of biosensor parameters, namely the thickness of the enzyme layer and the outer substrate concentration, on the response of the biosensor and it's sensitivity. The performed calculations showed a complex influence of the substrate and hydrogen peroxide concentrations.
\end{abstract}

\section{INTRODUCTION}

Biosensors are analytical devices converting a biochemical recognition reaction into a measurable effect (Scheller and Schubert, 1992; Turner et al., 1987). They are applied widely to monitor chemical substances in the medicine, food technology and the environmental industry (Wollenberger et al., 1997). Optical biosensors based on the fluorescence detection generate a photoluminescent signal indicative of target analyte binding ( $\mathrm{Li}-$ gler and Taitt, 2002). Fluorescence emission is then detected and converted into an analytical signal (Knopf and Bassi, 2007). They have been used for the analysis of many chemical and biological substances, especially in cases limited by low analyte concentrations (Atwood and Steed, 2004).

The mathematical model of optical biosensors has been very recently developed (Baronas et al., 2007a). However, the model was based upon absorbance measurements. In this report, a mathematical model for fluorescent biosensors is described. The model is used to predict the response of biosensor with varying physical and chemical characteristics.

\section{MATHEMATICAL MODEL}

The biochemical reaction of substrate $(\mathrm{S})$ with enzyme peroxidase $(\mathrm{E})$ in the presence of hydrogen peroxide
$\left(\mathrm{H}_{2} \mathrm{O}_{2}\right)$ to form the product $(\mathrm{P})$ may be expressed as

$$
\begin{aligned}
& \mathrm{E}+\mathrm{H}_{2} \mathrm{O}_{2} \stackrel{k_{1}}{\longrightarrow} \mathrm{I}+\mathrm{H}_{2} \mathrm{O}, \\
& \mathrm{I}+\mathrm{S} \stackrel{k_{2}}{\longrightarrow} \mathrm{E}+\mathrm{P},
\end{aligned}
$$

where $\mathrm{I}$ and $\mathrm{H}_{2} \mathrm{O}$ are the compound and water, respectively, $k_{1}$ and $k_{2}$ are the reaction rates.

The product $(\mathrm{P})$ emits light and therefore the response of the biosensor increases during the reaction as the product forms. The fluorescence of the product $(\mathrm{P})$ is proportional to the concentration of the analyte $(\mathrm{S})$ being measured. (Vo-Dinh, 2003).

Assuming the symmetrical geometry of the biosensor and homogeneous distribution of immobilized enzyme in the membrane, the mass transport and the reaction kinetics in the enzyme layer for the reaction scheme (1), (2) can be described by the following system of the reactiondiffusion equations $(0<x<d, t>0)$ :

$$
\begin{aligned}
& \frac{\partial S}{\partial t}=D_{S} \frac{\partial^{2} S}{\partial x^{2}}-k_{2} C S, \\
& \frac{\partial P}{\partial t}=D_{P} \frac{\partial^{2} P}{\partial x^{2}}+k_{2} C S, \\
& \frac{\partial H}{\partial t}=D_{H} \frac{\partial^{2} H}{\partial x^{2}}-k_{1} E H, \\
& \frac{\partial E}{\partial t}=-k_{1} E H+k_{2} C S, \\
& \frac{\partial C}{\partial t}=k_{1} E H-k_{2} C S,
\end{aligned}
$$

where $x$ and $t$ stand for space and time, $S(x, t), P(x, t)$, $H(x, t), E(x, t), C(x, t)$ are the substrate, product, hydrogen peroxide, enzyme and compound concentrations in the enzyme layer, $d$ is the thickness of the enzyme layer, $D_{S}, D_{P}, D_{H}$ are the diffusion coefficients. The enzyme and the formed compound are immobilized in the membrane and therefore there are no diffusion terms in the equations for the enzyme and compound.

Let $x=0$ represents the plate surface, while $x=d$ the boundary between the enzyme membrane and the bulk solution. The biosensor is initially immersed in a hydrogen peroxide solution. The operation of the biosensor starts when some substrate appears on the outer surface of the enzyme layer. This is used in the initial 
conditions $(t=0)$,

$$
\begin{aligned}
& P(x, 0)=C(x, 0)=0, \quad 0 \leq x \leq d, \\
& H(x, 0)=H_{0}, \quad 0 \leq x \leq d, \\
& E(x, 0)=E_{0}, \quad 0 \leq x \leq d, \\
& S(x, 0)=0, \quad 0 \leq x<d \\
& S(d, 0)=S_{0}
\end{aligned}
$$

where $E_{0}$ stands for the initial concentration of the enzyme in the enzyme membrane, $H_{0}$ is the hydrogen peroxide concentration in the bulk solution as well as the initial concentration in the enzyme membrane and $S_{0}$ is the substrate concentration in the bulk solution.

If the bulk solution is well-stirred and in powerful motion, then the diffusion layer $(0<x<d)$ remains at a constant thickness. In the bulk solution the concentration of the substrate, product and hydrogen peroxide remains constant $(t>0)$,

$$
S(d, t)=S_{0}, P(d, t)=0, H(d, t)=H_{0} .
$$

Assuming the impenetrable and unreactive plate surface, the mass flux of the species must vanish at this boundary,

$$
\left.\frac{\partial S}{\partial x}\right|_{x=0}=\left.\frac{\partial P}{\partial x}\right|_{x=0}=\left.\frac{\partial H}{\partial x}\right|_{x=0}=0 .
$$

By an extension of the Beer-Lambert law, the response of a fluorescent biosensor describing the intensity of fluorescence $F_{I}(t)$ at time $t$ may be obtained as follows (Baldini et al., 2006; Knopf and Bassi, 2007):

$$
F_{I}(t)=I_{0} \varphi\left(1-10^{-\varepsilon_{P} l_{e f} \bar{P}}\right), \quad l_{e f}=d,
$$

where $I_{0}$ stands for the intensity of excitation light, $\varphi$ is a quantum yield of fluorophore, $\varepsilon_{P}$ is the molar extinction coefficient of the product, $l_{e f}$ - effective thickness of the diffusion layer, $\bar{P}$ - the concentration of the product averaged through the entire diffusion layer. The quantum yield of the product fluorescence $\varphi$, defined as the ratio of the number of photons emitted to the number of photons absorbed, practically varies between 0.001 and 1. For organic compounds $\varepsilon_{P}$ varies between $10^{4}$ and $10^{2} \mathrm{~m}^{2} \mathrm{~mol}^{-1}$.

The fluorescence $F_{I}(t)$ is almost linearly proportional to the averaged concentration of the product $\bar{P}$,

$$
F_{I}(t) \approx 2.303 I_{0} \varphi \varepsilon_{P} l_{e f} \bar{P},
$$

when the absorbance expressed as $\varepsilon_{P} l_{e f} \bar{P}$ is less than 0.1 (Vo-Dinh, 2003). Since the fluorescence intensity $F_{I}(t)$ is directly proportional to the intensity $I_{0}$ of the excitation light, the measured fluorescence $F(t)$ is usually reported in relative fluorescent units (RFU),

$$
F(t)=F_{I}(t) / I_{0} .
$$

We assume that the system (3)-(10) approaches a steady state as $t \rightarrow \infty$,

$$
F_{\infty}=\lim _{t \rightarrow \infty} F(t),
$$

where $F_{\infty}$ is the steady state fluorescence.

The high sensitivity of the biosensor is especially important for the rapid detection of the analyte (Scheller and Schubert, 1992; Turner et al., 1987). The sensitivity is defined as a gradient of the steady state fluorescence with respect to the substrate concentration. The fluorescence varies in orders of magnitude with the concentration of the substrate to be analyzed. Therefore we consider dimensionless expression of the sensitivity,

$$
B_{S}\left(S_{0}\right)=\frac{S_{0}}{F_{\infty}\left(S_{0}\right)} \times \frac{d F_{\infty}\left(S_{0}\right)}{d S_{0}}
$$

where $B_{S}$ stands for the dimensionless sensitivity of the biosensor, $F_{\infty}\left(S_{0}\right)$ is the steady state fluorescence calculated at the substrate concentration $S_{0}$ in the bulk solution.

\section{DIGITAL SIMULATION}

No analytical solution for the problem is known, therefore a numerical approximation method must be used. We applied the implicit finite difference technique to solve the boundary value problem (3)-(10) (Britz, 2005; Samarskii, 2001). A uniform discrete grid was constructed in both directions $x$ and $t$. The resulting systems of linear algebraic equations were solved efficiently because of the tridiagonality of their matrices. The computational model was implemented in Java programming language (Moreira et al., 2000).

We define the steady state fluorescence $F_{R}$ of the biosensor as the fluorescence intensity calculated at the response time $T_{R}$,

$$
\begin{aligned}
& F_{R}=F\left(T_{R}\right) \approx F_{\infty}, \\
& T_{R}=\min _{j>0, F_{j}>0}\left\{\tau_{j}: \frac{F_{j}-F_{j-1}}{F_{j} \tau}<\varepsilon\right\},
\end{aligned}
$$

where $\tau$ is the size of time step. We used $\varepsilon=10^{-5}$ for the calculations.

Unless otherwise stated, we used the following values of the parameters:

$$
\begin{aligned}
& D_{S}=D_{P}=D_{H}=300 \mu \mathrm{m}^{2} \mathrm{~s}^{-1}, \\
& k_{1}=7.1 \times 10^{6} \mathrm{~s}^{-1} \mathrm{M}^{-1}, \\
& k_{2}=2 \times 10^{7} \mathrm{~s}^{-1} \mathrm{M}^{-1}, \\
& \varphi=0.01, \quad d=1 \mu \mathrm{m}, \\
& \varepsilon_{P}=10^{3} \mathrm{~m}^{2} \mathrm{~mol}^{-1} .
\end{aligned}
$$

The dynamics of the biosensor response having four different membrane thicknesses $d$ is presented in Fig. 1 . The steady state fluorescence appears with some delay corresponding to different thicknesses of the enzyme layers. The influence of the enzyme membrane thickness on the biosensor response was explored in greater detail (Baronas et al., 2003). 


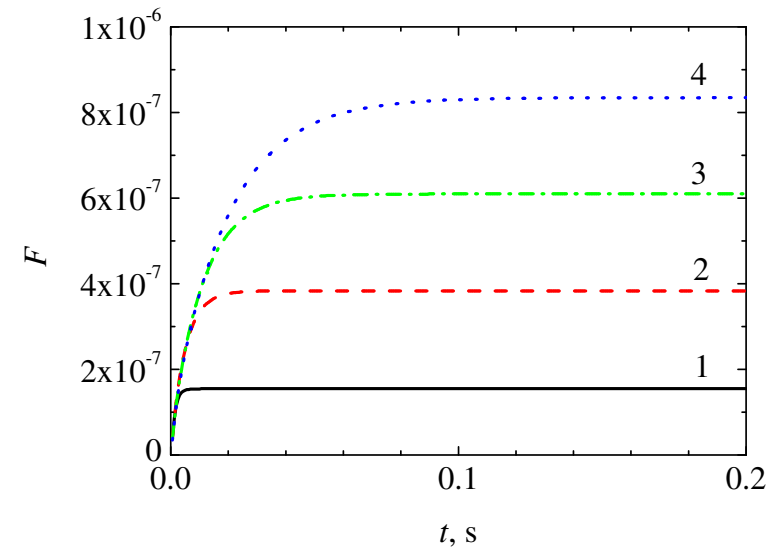

Figure 1: The dynamics of the fluorescence $F$ at four enzyme layer thicknesses $d$ : 1 (1), 2 (2), 3 (3), 4 (4) $\mu \mathrm{m}$, $S_{0}=100 \mu \mathrm{M}, E_{0}=1 \mathrm{nM}, H_{0}=1 \mathrm{mM}$

\section{RESULTS AND DISCUSSION}

Distributions of the concentrations of the substrate, product, hydrogen peroxide, complex and enzyme peroxidase in the enzyme membrane for an optical biosensor were given in a previous paper (Baronas et al., 2007a). By changing the input parameters we analyze the behavior of the response and sensitivity of the fluorescent biosensor.

\section{The Effect of the Thickness of the Enzyme Layer}

The effect of the thickness of the enzyme layer on the response of amperometric as well as potentiometric biosensors has been studied recently (Baronas et al., 2003, 2007b; Scheller and Schubert, 1992; Turner et al., 1987). Fig. 2 shows the dependence of the dimensionless steady state fluorescence $F_{R}$ on the thickness of enzyme membrane.

In the case of fluorescent biosensors, thickening the enzyme membrane causes the increase of the product concentration and therefore light is emitted to a greater extent. The response of the biosensor mostly depends on the outer concentration $S_{0}$ of the substrate (see curves 2 and 3 in Fig. 2). When the enzyme layer is relatively thick $(d>1 \mu \mathrm{m})$, the used variations of enzyme and hydrogen peroxide have similar effect on the fluorescence (see curves 1, 4, 5, 6 and 7). This can be explained by production of the same amount of the compound I, limited by the equation (1).

The effect of the enzyme layer thickness on the biosensor dimensionless sensitivity $B_{S}$ is shown in Fig. 3 . The sensitivity of the biosensor increases extending the enzyme layer. With exception of curves 2 and 7, the high values of sensitivity $\left(B_{S}>0.8\right)$ are observed within specified interval. At higher outer substrate concentration $S_{0}$ the enzyme becomes saturated and cannot respond effectively to the change of the substrate concentration (curve 2 ). The almost similar results were obtained at lower

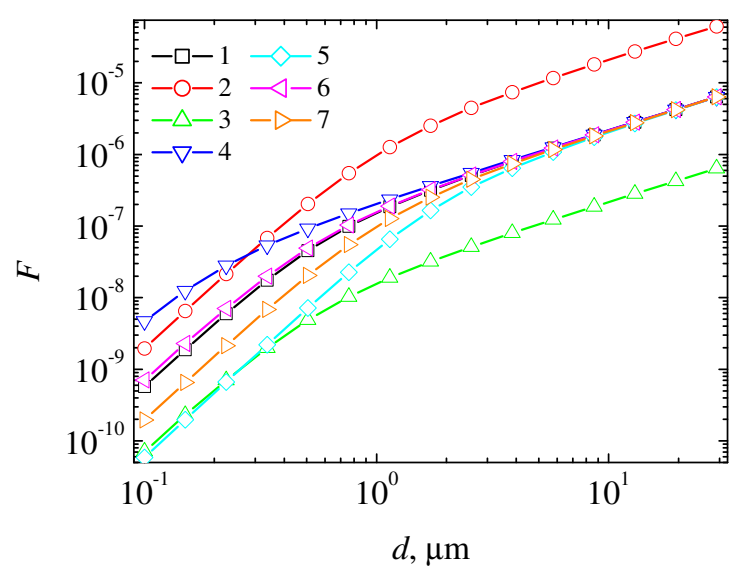

Figure 2: Dependence of the dimensionless steady state fluorescence $F_{R}$ on the thickness $d$ of the enzyme membrane at three substrate concentrations $S_{0}: 10$ (3), 100 $(1,4,5,6,7), 1000$ (2) $\mu \mathrm{M}$, three enzyme concentrations $E_{0}: 0.1(5), 1(1,2,3,6,7), 10(4) \mathrm{nM}$ and three hydrogen peroxide concentrations $H_{0}: 0.1$ (7), $1(1,2,3,4,5)$, $10(6) \mathrm{mM}$

concentration of hydrogen peroxide $H_{0}$ (curve 7). The sensitivity is significantly reduced because of the lower concentration of the formed compound I in comparison with the concentration of the substrate. In both cases, the sensitivity $B_{S}$ of the biosensor can be greatly enhanced by increasing the thickness of the enzyme layer.

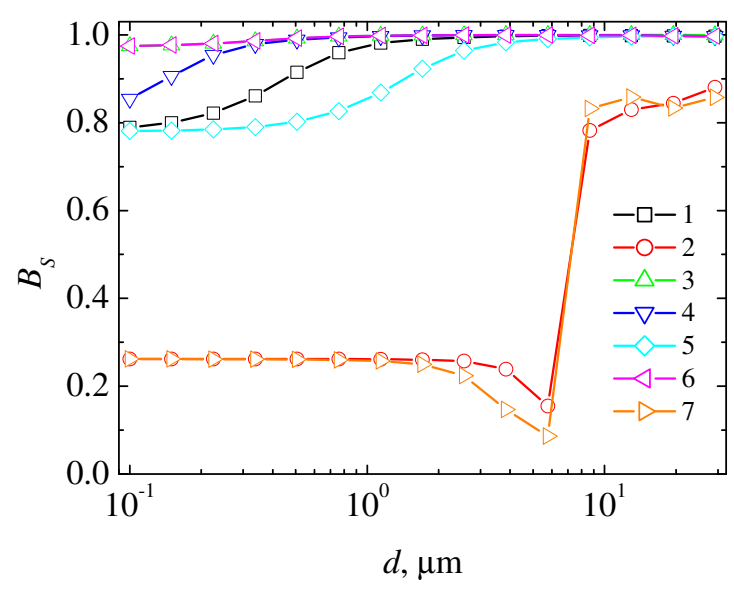

Figure 3: Dependence of the dimensionless sensitivity $B_{S}$ on the thickness $d$ of the enzyme membrane. The parameters and notation are the same as in Fig. 2

\section{The Effect of the Outer Substrate Concentration}

The effect of outer substrate concentration $S_{0}$ on the dimensionless steady state fluorescence $F_{R}$ is depicted in Fig. 4. The approximately linear curves of the fluorescence $F_{R}$ increase asymptotically approaching the steady 
states. A variation in the initial concentration $E_{0}$ of the enzyme affects the fluorescence, but has no effect on the limit of linearity of the calibration curve (see curves 2 and 3). However, the initial concentration $H_{0}$ of hydrogen peroxide have an effect upon the dynamic range of the calibration curves (curves 4 and 5). An increase of the concentration $H_{0}$ expands the limit of linearity and wise versa.

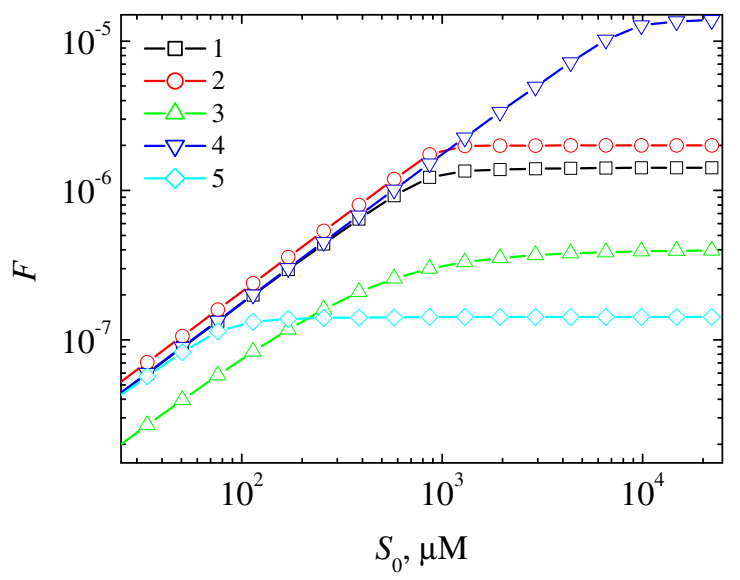

Figure 4: Dependence of the dimensionless steady state fluorescence $F_{R}$ on the substrate concentration in the bulk solution $S_{0}$ at three concentrations $E_{0}$ of the enzyme: $0.1(3), 1(1,4,5), 10(2) \mathrm{nM}$ and three concentrations $H_{0}$ of the hydrogen peroxide: $0.1(5), 1(1,2,3)$, 10 (4) $\mathrm{mM}$

The dimensionless sensitivity $B_{S}$ is markedly reduced as the concentration of the substrate $S_{0}$ increases (see Fig. 5). The change of the initial enzyme concentration $E_{0}$ has slight effect on the biosensor sensitivity (curves 2 and 3). However, the lower concentration of enzyme (curve 3) causes an unacceptable sensitivity at $S_{0} \approx>$ $1.1 \times 10^{2} \mu \mathrm{M}$. The high values of the sensitivity up to $S_{0} \approx 10^{4} \mu \mathrm{M}$ can be observed with the increased initial concentration $H_{0}$ of hydrogen peroxide (curve 4).

\section{CONCLUSIONS}

The developed mathematical model (3)-(10) of a peroxidase-based fluorescent biosensor can be used for a digital simulation and an investigation of the biosensor response.

By providing a relatively thick enzyme layer a higher sensitivity and fluorescence values can be obtained. However, a great care must be taken in cases of high outer substrate and low initial hydrogen peroxide concentrations (Fig. 3).

The higher sensitivity of the fluorescent biosensor can be achieved by increasing the concentration of the hydrogen peroxide (Fig. 5).

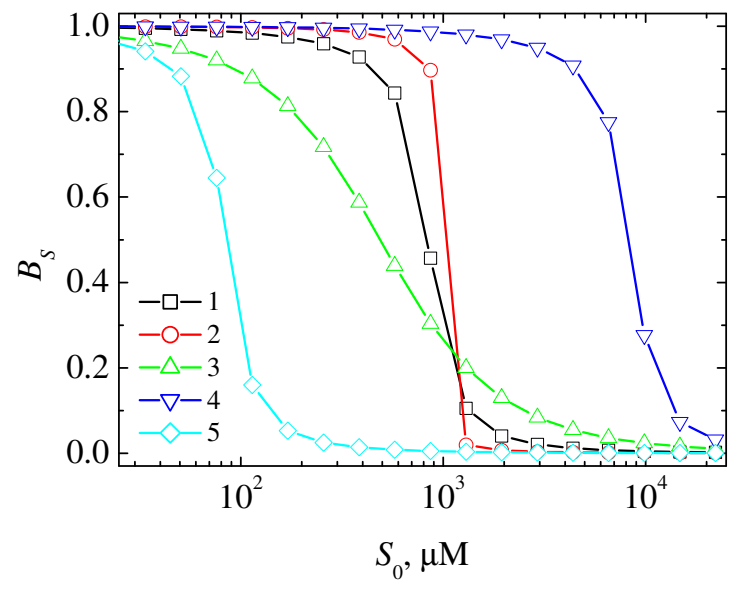

Figure 5: Dependence of the dimensionless sensitivity $B_{S}$ on the substrate concentration $S_{0}$ in the bulk solution. The parameters and notation are the same as in Fig. 4

\section{ACKNOWLEDGEMENTS}

The authors express sincere gratitude to prof. Feliksas Ivanauskas and prof. Juozas Kulys for their valuable contribution to modelling of biosensors.

\section{References}

Atwood, J. L. and J. W. Steed. (2004). Encyclopedia of Supramolecular Chemistry. Marcel Dekker, New York.

Baldini, F.; A. N. Chester; J. Homola; and S. Martellucci. (2006). Optical Chemical Sensors. Springer, Amsterdam.

Baronas, R.; E. Gaidamauskaite; and J. Kulys. (2007). ”Modelling a Peroxidase-based Optical Biosensor". Sensors, 7(11):2723-2740.

Baronas, R.; F. Ivanauskas; and J. Kulys. (2003). 'The influence of the enzyme membrane thickness on the response of amperometric biosensors". Sensors, 3(7):248-262.

Baronas, R.; F. Ivanauskas; and J. Kulys. (2007). "Computational modelling of the behaviour of potentiometric membrane biosensors". Journal of Mathematical Chemistry, 42(3):321-336.

Britz, D. (2005). Digital Simulation in Electrochemistry. Springer-Verlag, Berlin.

Knopf, G. K. and A. S. Bassi. (2007). Smart Biosensor Technology. CRC Press, New York.

Ligler, F. S. and C. R. Taitt. (2002). Optical Biosensors: Present and Future. Elsevier Science, Amsterdam.

Moreira, J. E.; S. P. Midkiff; M. Gupta; P. V. Artigas; M. Snir; and R. D. Lawrence. (2000). "Java programming for high performance numerical computing". IBM Systems J., 39:2156.

Samarskii, A. A. (2001). The Theory of Difference Schemes. Marcel Dekker, New York-Basel. 
Scheller, F. and F. Schubert. (1992). Biosensors. Elsevier, Amsterdam.

Turner, A. P. F.; I. Karube; and G. S. Wilson. (1987). Biosensors: Fundamentals and Applications. Oxford University Press, Oxford.

Vo-Dinh, T. (2003). Biomedical Photonics Handbook. CRC Press, New York.

Wollenberger, U.; F. Lisdat; and F. W. Scheller. (1997). Frontiers in Biosensorics 2: Practical Applications. Birkhauser Verlag, Basel.

\section{AUTHOR BIOGRAPHIES}

EVELINA GAIDAMAUSKAITE was born in Vilnius, Lithuania. She is currently a PhD student at Vilnius University. She received her BSc and MSc degrees in Computer Science in 2004 and 2006, respectively from Vilnius University. Her research interests are in computational modelling of biosensors. Her e-mail is: evelina.gaidamauskaite@mif.vu.lt.

ROMAS BARONAS was born in 1959 in Kybartai, Lithuania. He is a professor and serves as chair of the Department of Software Engineering at Vilnius University. Prof. Baronas received his MSc degree in Applied Mathematics in 1982 and then obtained his $\mathrm{PhD}$ degree in Computer Science in 2000 from the Vilnius University. He also joined the Software Engineering Department at the Institute of Mathematics and Informatics. His teaching and research interests lie in the areas of database systems and computational modelling of biochemical processes. His e-mail address is: romas.baronas@mif.vu.lt and his personal Web-page can be found at http://www.mif.vu.lt/ baronas. 